Acta Crystallographica Section D

\section{Biological Crystallography}

ISSN 1399-0047

\section{Yung-Lin Wang, ${ }^{a, b} \neq$ Sih-Yao Chow, ${ }^{b} \ddagger$ Yi-Ting Lin, ${ }^{b}$ Yu-Chiao Hsieh, $^{b}$ Guan-Chiun Lee ${ }^{\mathrm{c} *}$ and Shwu-Huey Liaw ${ }^{a, b, d}$ *}

anstitute of Biochemistry and Molecular Biology, National Yang-Ming University, Taipei 11221, Taiwan, ${ }^{\mathbf{b}}$ Department of Life Sciences and Institute of Genome Sciences, National Yang-Ming University, Taipei 11221, Taiwan, ' Department of Life Sciences, National Taiwan Normal University, Taipei 11677, Taiwan, and d Department of Medical Research and Education, Taipei Veterans General Hospital, Taipei 11217, Taiwan

₹ These authors contributed equally to this work.

Correspondence e-mail: gclee@ntnu.edu.tw, shliaw@ym.edu.tw

\title{
Structures of trehalose synthase from Deinococcus radiodurans reveal that a closed conformation is involved in catalysis of the intramolecular isomerization
}

Trehalose synthase catalyzes the simple conversion of the inexpensive maltose into trehalose with a side reaction of hydrolysis. Here, the crystal structures of the wild type and the N253A mutant of Deinococcus radiodurans trehalose synthase (DrTS) in complex with the inhibitor Tris are reported. DrTS consists of a catalytic $(\beta / \alpha)_{8}$ barrel, subdomain B, a C-terminal $\beta$ domain and two TS-unique subdomains (S7 and S8). The C-terminal domain and S8 contribute the majority of the dimeric interface. DrTS shares high structural homology with sucrose hydrolase, amylosucrase and sucrose isomerase in complex with sucrose, in particular a virtually identical activesite architecture and a similar substrate-induced rotation of subdomain B. The inhibitor Tris was bound and mimics a sugar at the -1 subsite. A maltose was modelled into the active site, and subsequent mutational analysis suggested that Tyr213, Glu320 and Glu324 are essential within the +1 subsite for the TS activity. In addition, the interaction networks between subdomains B and S7 seal the active-site entrance. Disruption of such networks through the replacement of Arg148 and Asn253 with alanine resulted in a decrease in isomerase activity by $8-9$-fold and an increased hydrolase activity by $1.5-$ 1.8-fold. The N253A structure showed a small pore created for water entry. Therefore, our DrTS-Tris may represent a substrate-induced closed conformation that will facilitate intramolecular isomerization and minimize disaccharide hydrolysis.

\section{Introduction}

Trehalose is a nonreducing disaccharide formed by an $\alpha, \alpha-1,1-$ linkage of two glucose molecules and displays exceptional stability. This sugar is used by a wide variety of organisms for a variety of key functions such as as an energy/carbon source, for signalling and for protein/membrane protection, as well as being used as a bacterial cell-wall component (Elbein et al., 2003). Trehalose plays an essential role in Mycobacterium tuberculosis metabolism and thus enzymes involved in the biosynthesis of this sugar serve as possible drug targets (Hunter et al., 2006). In addition, trehalose stabilizes a wide variety of biomolecules and hence has been used widely in the food, cosmetics, medical and biotechnological industries. Trehalose synthase (TS) catalyzes the reversible conversion of the inexpensive maltose into trehalose in the absence of any coenzyme. This enzymatic synthesis has a number of advantages, namely a simple reaction, high substrate specificity and low cost; hence, this could easily form a potential method for the production of trehalose.

TS belongs to glycoside hydrolase family 13 (GH13), which includes a diverse range of carbohydrate-metabolizing
Received 4 July 2014 Accepted 13 October 2014

PDB references: DrTS, wild type, 4tvu; N253A mutant, $4 \mathrm{wf7}$ 
Table 1

Statistics of data collection and structural refinement.

Values in parentheses are for the highest resolution shell.

\begin{tabular}{|c|c|c|}
\hline & Wild type & $\mathrm{N} 253 \mathrm{~A}$ \\
\hline PDB code & 4 tvu & $4 \mathrm{wf} 7$ \\
\hline \multicolumn{3}{|l|}{ Data collection } \\
\hline Space group & $P 2_{1}$ & $P 2_{1} 2_{1} 2_{1}$ \\
\hline $\begin{array}{l}\text { Unit-cell parameters } \\
\left(\AA{ }^{\circ},{ }^{\circ}\right)\end{array}$ & $\begin{array}{l}a=95.61, b=195.90 \\
\quad c=130.99 \\
\alpha=\gamma=90, \beta=92.89\end{array}$ & $\begin{array}{c}a=96.25, b=133.71, \\
c=196.61, \\
\alpha=\beta=\gamma=90\end{array}$ \\
\hline Resolution $(\AA)$ & $30-2.70(2.80-2.70)$ & $20-2.21(2.29-2.21)$ \\
\hline Observed reflections & 462831 (38844) & 759037 (71092) \\
\hline Unique reflections & 128557 (11771) & $129348(12695)$ \\
\hline Completeness (\%) & $97.7(89.4)$ & $99.9(99.7)$ \\
\hline Multiplicity & $3.6(3.3)$ & $5.9(5.6)$ \\
\hline$\langle I / \sigma(I)\rangle$ & $13.1(2.9)$ & $12.6(2.3)$ \\
\hline$R_{\text {merge }} \dagger(\%)$ & $9.6(40.8)$ & $15.1(78.7)$ \\
\hline \multicolumn{3}{|l|}{ Refinement } \\
\hline Resolution $(\AA)$ & $30-2.7(2.77-2.70)$ & $20-2.21(2.27-2.21)$ \\
\hline Reflections $[F>0 \sigma(F)]$ & $122065(8113)$ & $119863(8156)$ \\
\hline$R_{\text {cryst }} \ddagger(\%)$ & $19.4(28.2)$ & $16.0(22.6)$ \\
\hline$R_{\text {free }} \S(\%)$ & $23.6(31.9)$ & $20.0(26.3)$ \\
\hline \multicolumn{3}{|l|}{ R.m.s. deviations } \\
\hline Bond lengths $(\AA)$ & 0.01 & 0.01 \\
\hline Bond angles $\left({ }^{\circ}\right)$ & 1.42 & 1.41 \\
\hline \multicolumn{3}{|l|}{ Mean $B$ value $\left(\AA^{2}\right)$} \\
\hline Protein & 38.0 & 33.0 \\
\hline Metal ions & 27.1 & 26.7 \\
\hline Tris & 32.5 & 35.9 \\
\hline Water & 24.9 & 37.3 \\
\hline \multicolumn{3}{|c|}{ Ramachandran analysis $(\%)$} \\
\hline Favoured & 97.4 & 97.4 \\
\hline Allowed & 2.6 & 2.6 \\
\hline Disallowed & 0 & 0 \\
\hline
\end{tabular}

$\dagger R_{\text {merge }}=\sum_{h k l} \sum_{i}\left|I_{i}(h k l)-\langle I(h k l)\rangle\right| / \sum_{h k l} \sum_{i} I_{i}(h k l)$, where $I_{i}(h k l)$ is the average intensity value of the equivalent reflections. $\$ R_{\text {cryst }}=\sum_{h k l}|| F_{\text {obs }}|-| F_{\text {calc }}|| / \sum_{h k l}\left|F_{\text {obs }}\right|$, where $F_{\text {obs }}$ and $F_{\text {cac }}$ are the observed and the calculated structure factors, respectively. $\& R_{\text {free }}$ was calculated using $5 \%$ of data that were randomly excluded from refinement. ๆ The Ramachandran analysis was performed by MolProbity (Chen et al., 2010).

enzymes and has been further divided into 35-37 subfamilies (Stam et al., 2006; Cantarel et al., 2009). The GH13 enzymes share a catalytic $(\beta / \alpha)_{8}$ barrel as their structurally conserved core together with a few conserved regions; this is despite their having a relatively low overall sequence identity (Svensson, 1988). They also include a variable number of member-unique subdomains that as yet remain without any known function. As for other GH13 enzymes, mechanistic analysis of TS from M. smegmatis (MsTS) demonstrated that this enzyme employs a double-displacement mechanism with a covalent glycosylenzyme intermediate (Zhang et al., 2011). Further kinetic studies have suggested an intramolecular mechanism within an enclosed active site and that the conformational changes of the protein are rate-limiting. However, the recently published structures of MsTS and M. tuberculosis TS (MtTS) are nearly identical and show an inactive open conformation (Caner et al., 2013; Roy et al., 2013). In the present study, we have determined crystal structures of the wild type and the N253A mutant of TS from Deinococcus radiodurans (DrTS) that reveal a closed conformation for intramolecular isomerization. The available TS structures demonstrate that the ratedetermining conformational changes are mediated mainly by TS-unique insertions that are involved in modulating the opening and closing of the active site. Such conformational changes that involve the intervention of member-unique subdomains during enzyme catalysis can also be observed in some other GH13 members.

\section{Materials and methods}

\subsection{Protein preparation and sedimentation analysis}

Mutational analysis was carried out using a QuikChange site-directed mutagenesis kit (Stratagene). The recombinant wild-type and mutant DrTS were expressed in Escherichia coli BL21 (DE3) cells using the pET-23a(+) vector (Qiagen; Wang et al., 2007). Luria broth cultures were grown at $310 \mathrm{~K}$ and induced with $0.4 \mathrm{~m} M$ isopropyl $\beta$-D-1-thiogalactopyranoside at $289 \mathrm{~K}$ for $16 \mathrm{~h}$. Cell pellets were resuspended in lysis buffer consisting of $20 \mathrm{~m} M$ sodium phosphate, $500 \mathrm{mM} \mathrm{NaCl} \mathrm{pH} 7.4$ and lysed using a French press. After the removal of cellular debris by centrifugation at $39000 \mathrm{~g}$ at $277 \mathrm{~K}$ for $30 \mathrm{~min}$, the crude extract was applied onto a $5 \mathrm{ml}$ nickel-nitrilotriacetic acid column (Qiagen). After washing with 20-60 mM imidazole, the protein was eluted with $250 \mathrm{~m} M$ imidazole and dialyzed against $20 \mathrm{~m} M$ HEPES pH 7.5, $100 \mathrm{~m} M \mathrm{NaCl}, 1 \mathrm{~m} M$ dithiothreitol at $277 \mathrm{~K}$. The molecular mass in solution was estimated using a Beckman-Coulter XL-A analytical ultracentrifuge with an An50Ti rotor. Sedimentation-velocity centrifugation was performed at $293 \mathrm{~K}$ and $42000 \mathrm{rev} \mathrm{min}{ }^{-1}$ with double-sector epon charcoal-filled centrepieces. The absorption of the cells at $280 \mathrm{~nm}$ was scanned every $5 \mathrm{~min}$ for $5 \mathrm{~h}$ and the data were fitted to a continuous $c(s)$ distribution model using SEDFIT (Schuck et al., 2002). All size and shape distributions were analyzed at a confidence level of $p=0.95$ by maximal entropy regularization and a resolution $N$ of 200 with sedimentation coefficients between 0 and $20 \mathrm{~S}$.

\subsection{Activity assay}

The isomerase and hydrolase activities of DrTS were determined by measuring the amount of trehalose and glucose produced from maltose, respectively (Wang et al., 2007). The inhibitory effect of Tris on the DrTS isomerase activity was determined in an assay solution by the addition of $10 \mu \mathrm{g}$ purified DrTS to $100 \mu \mathrm{l} 50 \mathrm{~m} M$ maltose solution in $20 \mathrm{mM}$ sodium phosphate $\mathrm{pH} 7.4$ at $20^{\circ} \mathrm{C}$ for $2 \mathrm{~h}$. For the mutants, the TS activity was assayed in a reaction mixture consisting of $200 \mu 10.25 \mathrm{mg} \mathrm{ml}^{-1}$ purified DrTS and $800 \mu 125 \mathrm{mM}$ maltose solution in $20 \mathrm{mM}$ sodium phosphate $\mathrm{pH} 7.4$ at $20^{\circ} \mathrm{C}$ for $2 \mathrm{~h}$. The activity assay for each mutant was carried out in quadruplicate. The reaction was terminated by heating the mixture in boiling water for $15 \mathrm{~min}$. The amount of maltose, trehalose and glucose in each reaction mixture was measured using a high-performance liquid-chromatography system (Schambeck SFD 2100) equipped with a refractive-index detector (SFD, RI 2000) at a flow rate of $1 \mathrm{ml} \mathrm{min}^{-1}$. A carbohydrate-analysis column $(6.0 \times 150 \mathrm{~mm}$, Shodex SZ5532) equilibrated with $75 \%$ acetonitrile, $24 \%$ Milli-Q water and 1\% formic acid was used. One unit of the isomerase or hydrolase activity was defined as the amount of enzyme that catalyzes the formation of $1 \mathrm{nmol}$ of trehalose or glucose per minute. 


\subsection{Structure analysis}

The initial crystallization screening was performed with screening kits using the hanging-drop vapour-diffusion method at $288 \mathrm{~K}$. The hanging drops were mixtures of $2 \mu \mathrm{l}$ reservoir solution and $2 \mu \mathrm{l}$ protein solution. Crystals of the wild-type protein were grown in 9\% PEG 4000, $0.2 \mathrm{M}$ sodium acetate trihydrate, $0.3 \mathrm{M}$ Tris- $\mathrm{HCl} \mathrm{pH} 8.5$ using a protein solution at $30 \mathrm{mg} \mathrm{ml}^{-1}$ in $6-8$ weeks. The N253A mutant crystals were obtained in $11 \%$ PEG 4000, $0.2 \mathrm{M}$ sodium acetate trihydrate, $0.3 \mathrm{M}$ Tris- $\mathrm{HCl} \mathrm{pH} 8.5,5 \%$ glycerol using a protein solution at $60 \mathrm{mg} \mathrm{ml}^{-1}$ in two weeks. X-ray diffraction data were collected and processed on beamlines BL13B1 and BL15A1 at NSRRC, Hsinchu, Taiwan. The wild-type and N253A crystals belonged to space groups $P 2_{1}$ and $P 2_{1} 2_{1} 2_{1}$, respectively. The structures were determined by the molecularreplacement method using the MsTS structure as a template (Caner et al., 2013); the search models were generated by MODELLER (Šali \& Blundell, 1993) and the phase problem was solved using Phaser (McCoy et al., 2007). Further refinement was performed with Coot (Emsley et al., 2010) and REFMAC5 (Murshudov et al., 2011), and the Ramachandran analysis was performed using MolProbity (Chen et al., 2010). Noncrystallographic symmetry restraints were applied in structural refinement of the wild type but not in that of the N253A mutant. The statistics of data collection and refinement are summarized in Table 1. Figures were generated using PyMOL (DeLano, 2002).

\section{Results and discussion}

\subsection{The DrTS structure}

Analytical ultracentrifugation experiments were able to clearly demonstrate that DrTS exists as a dimer in solution as well as in the crystal form, with each asymmetric unit containing two and four DrTS dimers in space groups $P 2_{1} 2_{1} 2_{1}$ and $P 2_{1}$, respectively (Figs. 1 and $2 a$ ). Clear electron density was observed covering the entire DrTS molecule, except for residues $1-5$ and the vector linkers. The four or eight subunits in each asymmetric unit did not display significant differences, with root-mean-square deviations of $0.3-0.4 \AA$ for all $\mathrm{C}^{\alpha}$ atoms. As in other GH13 members, each DrTS protomer mainly consists of a catalytic $(\beta / \alpha)_{8}$ barrel $(\beta 1-\beta 8, \alpha 1-\alpha 8$ and $\alpha 6^{\prime}$ ) as the central core and a C-terminal $\beta$-sandwich (residues 463-552; domain C) (Fig. 2b). Domain $C$ is made up of two antiparallel $\beta$-sheets with five $(\mathrm{C} \beta 1-\mathrm{C} \beta 3, \mathrm{C} \beta 5$ and $\mathrm{C} \beta 7)$ and two $(\mathrm{C} \beta 4$ and $\mathrm{C} \beta 6) \beta$-strands. This region is tightly connected to the $(\beta / \alpha)_{8}$ barrel through an extensive network of interactions consisting of nine hydrogen bonds and various hydrophobic patches involving 47 residues, which are mainly contributed by its five-stranded $\beta$-sheet and $\alpha 6, \alpha 6^{\prime}, \alpha 7$ and $\alpha 8$. In TSs, domain $\mathrm{C}$ has the greatest diverse in sequence; while DrTS shares $\sim 50 \%$ sequence identity with MtTS overall, they display only $\sim 25 \%$ sequence identity in domain $\mathrm{C}$.

Three additional subdomains are formed by long extrusion fragments inserted between $\beta 3$ and $\alpha 3$, between $\beta 7$ and $\alpha 7$, and between $\beta 8$ and $\alpha 8$ within the $(\beta / \alpha)_{8}$ barrel, and they are designated as subdomains B, S7 and S8, respectively (Fig. 2b). These subdomains are all loop-rich structures. Subdomain B (residues 105-184) is common to the GH13 family and consists of one short helix $(\mathrm{B} \alpha 1)$ and three antiparallel $\beta$-strands $(\mathrm{B} \beta 1-\mathrm{B} \beta 3)$. This subdomain strongly interacts with the $(\beta / \alpha)_{8}$ barrel by forming 16 hydrogen bonds and extensive hydrophobic contacts between 58 residues. On the other hand, subdomains S7 and S8 are unique to TS. Specifically, S7 (residues 315-361) contains a short helix $\left(\alpha 7^{\prime}\right)$, while S8 (residues 384-449) does not display any significant secondary structure. The $(\beta / \alpha)_{8}$ barrel contains the catalytic cleft, which consists of the triad Asp209-Glu251-Asp319 and various conserved substrate-binding residues. Domain $\mathrm{C}$ and subdomain S8 contribute to dimer formation, while subdomains B and S7 serve as a gateway for the opening and closing of the active site. These points will be discussed later.

In addition, many GH13 members contain metal ions, which may be involved in the maintenance of structural integrity (Kobayashi et al., 2011). In the $\alpha$-amylase from the human gut bacterium Bacteroides thetaiotaomicron (BtSusG) and MsTS, one $\mathrm{Mg}^{2+}$ and one $\mathrm{Ca}^{2+}$ ion were identified (Koropatkin \& Smith, 2010; Caner et al., 2013). In DrTS two similar metal ionbinding sites can also be observed and hence the same divalent cations have been modelled into the electron density (Fig. $2 b$ ). The $\mathrm{Mg}^{2+}$ site consists of the side chains of Asp24, Asn26, Asp28 and Asp32 and the main-chain O atom of Lys30, which form part of the consensus signature $\mathrm{D} X \mathrm{~N} X \mathrm{DG} X \mathrm{GD}$. Such a site is also found in some other GH13 members, for instance the similar calcium sites in Rhizobium sp. MX-45 sucrose
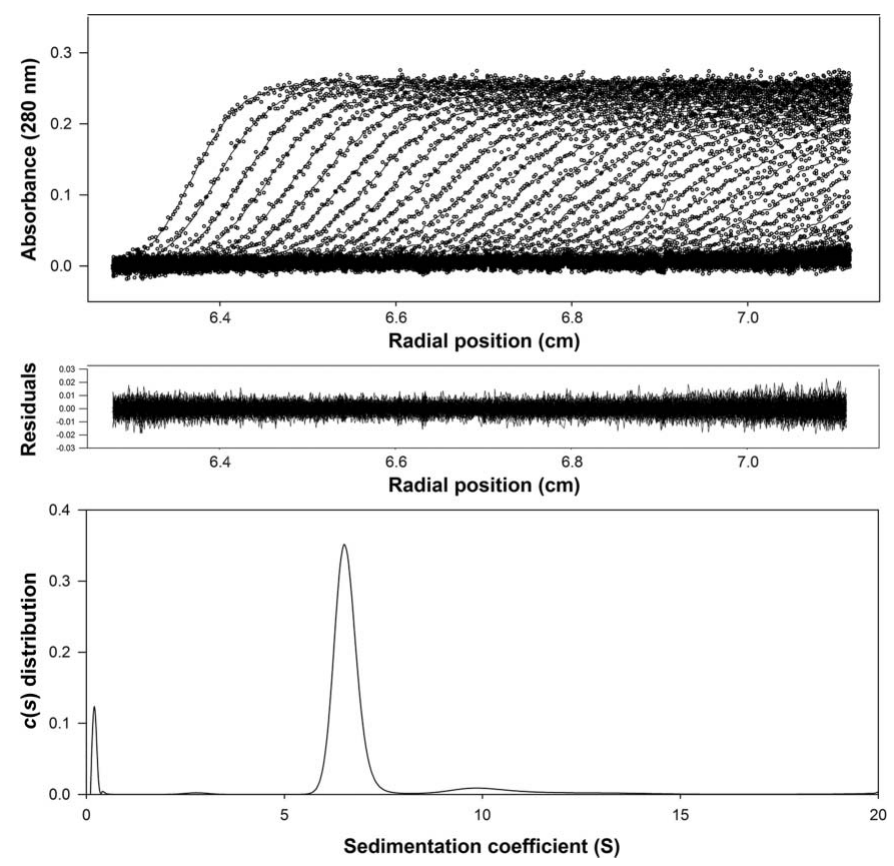

Figure 1

Sedimentation-velocity analysis of DrTS. The enzyme concentration was $0.1 \mathrm{mg} \mathrm{ml}^{-1}$ in $20 \mathrm{~m} M$ Tris- $\mathrm{HCl} \mathrm{pH} 7.5,100 \mathrm{mM} \mathrm{NaCl}$. The three panels represent the optical traces (top), the residuals of the model fitting (middle) and the sedimentation-coefficient distribution (bottom). The ultracentrifugation analysis demonstrated that DrTS exists as a dimer in solution. 
isomerase (trehalulose synthase; RhSI) and in yeast isomaltase (ScIM; Ravaud et al., 2007; Yamamoto et al., 2010). On the other hand, the $\mathrm{Ca}^{2+}$ site is coordinated by the side chains of Asn105, Asp179 and Glu216 and the backbone O atoms of Tyr213 and Leu214. Many $\alpha$-amylases contain a similar calcium site that involves the conserved asparagine and aspartate residues (Asn105 and Asp179 in DrTS; Janeček et al., 2014). Interestingly, in the closest structural neighbours of TS this site is occupied by a lysine, which replaces the ligand glutamate residue (Glu216 in DrTS).

\subsection{Closest structural neighbours}

A search using the DALI server (Holm \& Rosenström, 2010) revealed that DrTS shares the greatest amount of

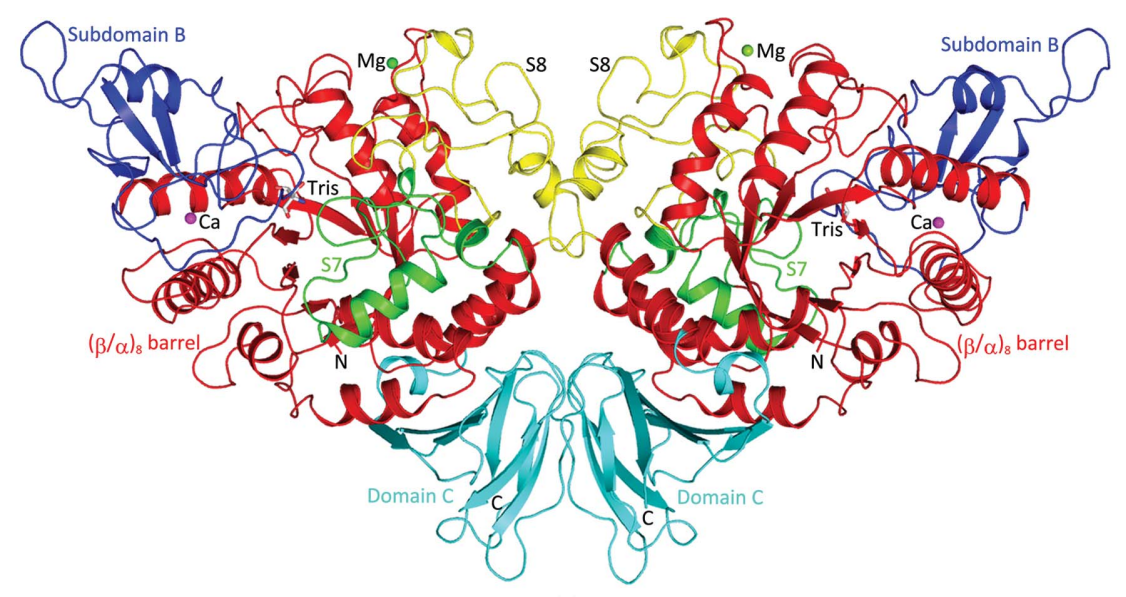

(a)
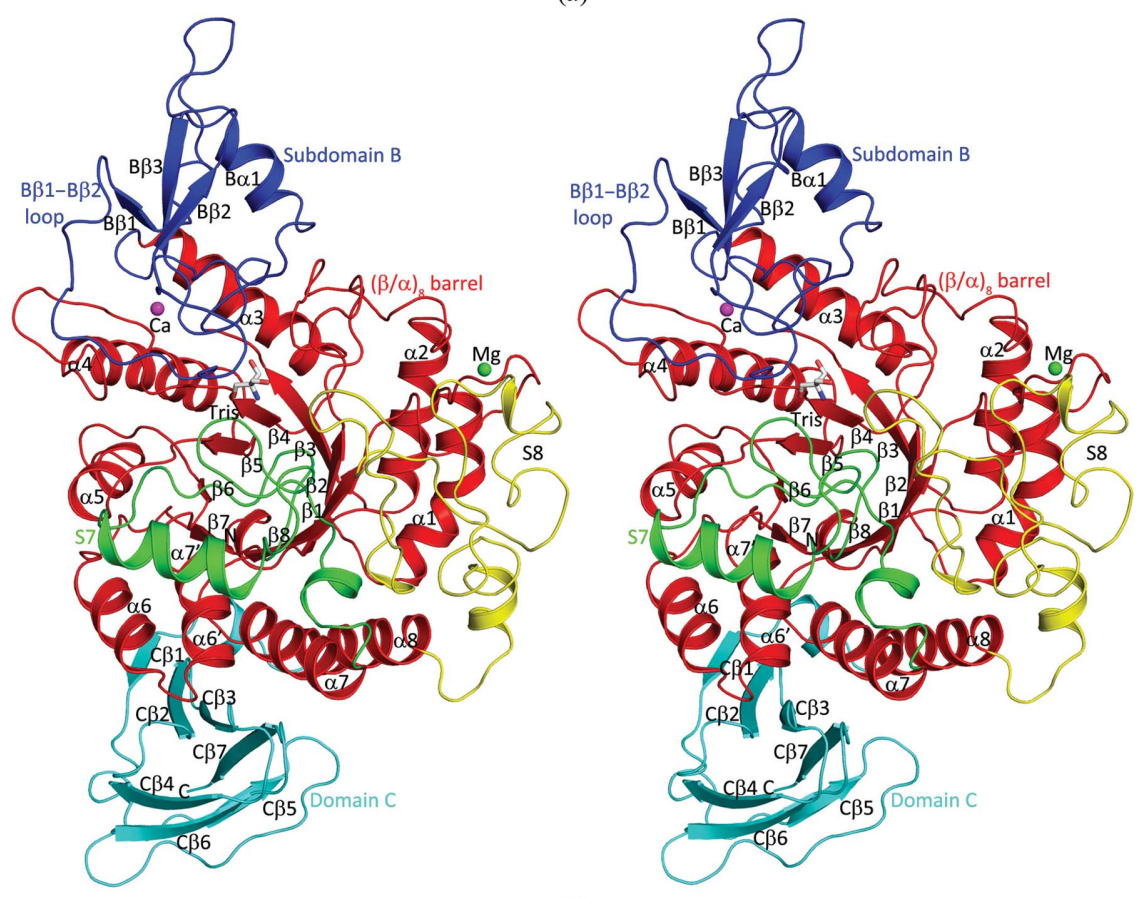

(b)

Figure 2

Ribbon views of the DrTS dimer $(a)$ and monomer $(b)$ structures. The protein consists of a $(\beta / \alpha)_{8}$ barrel (red), subdomain B (blue), domain C (cyan) and two TS-unique modules (S7 in green and $\mathrm{S} 8$ in yellow). The bound $\mathrm{Mg}^{2+}$ and $\mathrm{Ca}^{2+}$ ions are shown as green and magenta spheres, respectively, with the Tris molecule shown as a stick representation. The dimeric interfaces are formed mainly by domain C and S8 and have a total buried area of $\sim 3000 \AA^{2}$. structural similarity with MsTS, MtTS, Xanthomonas axonopodis sucrose hydrolase (XaSH), Neisseria polysaccharea amylosucrase (NpAS), RhSI, ScIM and BtSusG, with rootmean-square deviations (r.m.s.d.s) of $1.8 \AA$ (PDB entry 3zoa; $530 \mathrm{C}^{\alpha}$ atoms with $52 \%$ sequence identity), $2.1 \AA$ (PDB entry 4lxf; $524 \mathrm{C}^{\alpha}$ atoms with $53 \%$ identity), $1.7 \AA$ (PDB entry 3czk; $482 \mathrm{C}^{\alpha}$ atoms with $28 \%$ identity), $1.9 \AA$ (PDB entry $1 \mathrm{mw} 0 ; 485$ $\mathrm{C}^{\alpha}$ atoms with $28 \%$ identity), $2.3 \AA$ (PDB entry 2 pwe; $476 \mathrm{C}^{\alpha}$ atoms with $33 \%$ identity), $2.4 \AA$ (PDB entry $3 \mathrm{axh} ; 476 \mathrm{C}^{\alpha}$ atoms with $29 \%$ identity) and $2.6 \AA$ (PDB entry $3 \mathrm{k} 81 ; 458 \mathrm{C}^{\alpha}$ atoms with 31\% identity), respectively (Caner et al., 2013; Roy et al., 2013; Kim et al., 2008; Skov et al., 2002; Ravaud et al., 2007; Yamamoto et al., 2011; Koropatkin \& Smith, 2010; Fig. 3). The $(\beta / \alpha)_{8}$ barrel is the most superimposable part of the structure, as are parts of subdomain $\mathrm{B}$ and domain $\mathrm{C}$ (Figs. $3 a, 3 b$ and $3 c$ ). The most divergent parts of the $(\beta / \alpha)_{8}$ barrel are the member-unique modules inserted between $\beta 4$ and $\alpha 4$, between $\beta 6$ and $\alpha 6$, between $\beta 7$ and $\alpha 7$ (S7), and between $\beta 8$ and $\alpha 8$ (S8) (Figs. $3 a$ and $3 d$ ), which are located on the top of the active site and may modulate the size/shape and accessibility of the active site. In subdomain $\mathrm{B}$, the most diverse segment is the region between $\mathrm{B} \beta 1$ and $\mathrm{B} \beta 2$, the socalled $\mathrm{B} \beta 1-\mathrm{B} \beta 2$ loop (Fig. $3 b$ ), which lines one side of the active-site entrance and hence is involved in substrate access and binding. The member-unique insertions and subdomain $\mathrm{B}$ will be discussed later.

Domain $\mathrm{C}$ displays a distinct lack of significant sequence similarity. Superposition of domain $\mathrm{C}$ reveals that the fivestranded $\beta$-sheet is more superimposable, whereas the two-stranded $\beta$-sheet is more diverse (Fig. 3c). The former is tightly packed with the $(\beta / \alpha)_{8}$ barrel, while the latter may contribute to the functional diversity. For example, in TS this twostranded $\beta$-sheet makes up the dimer interface, while in a number of GH13 members this part may function as a carbohydratebinding module (Christiansen et al., 2009). In contrast to most GH13 members, which are monomeric, DrTS is dimeric, while MtTS forms a tetramer (Roy et al., 2013). The dimeric interfaces are mainly mediated by the TS-unique S8 and domain C (Fig. $2 a$ ). Owing to the sequence diversity of domain $\mathrm{C}$, the hydrogen bonds and hydrophobic contacts in the dimeric interfaces of DrTS and MtTS are distinct. This difference accounts for the various oligomer states. In DrTS, there are 26 direct hydrogen bonds between the protein atoms across the dimer interface, whereas there are only ten direct hydrogen bonds in MtTS. As a result, 

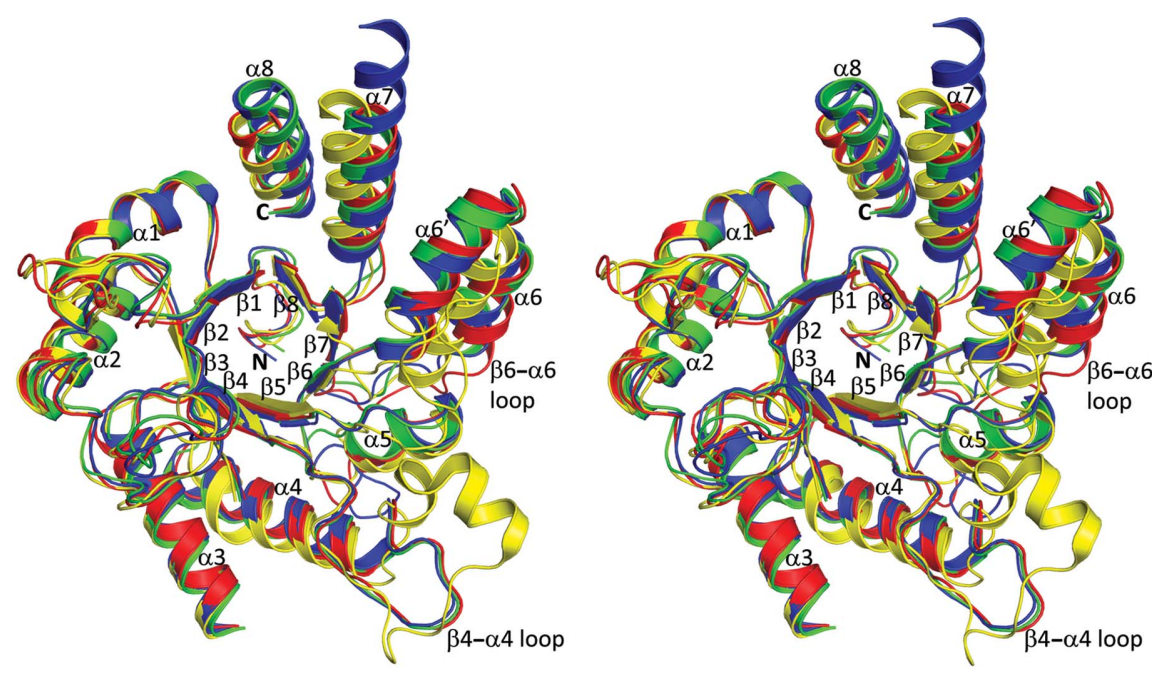

(a)

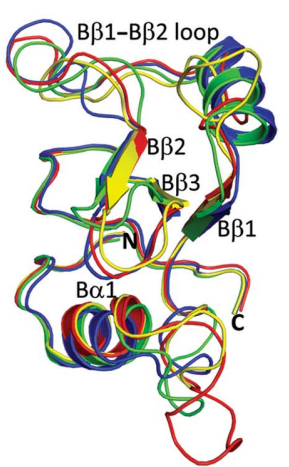

(b)

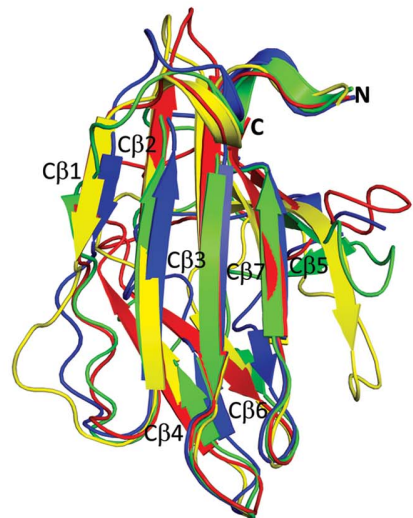

(c)

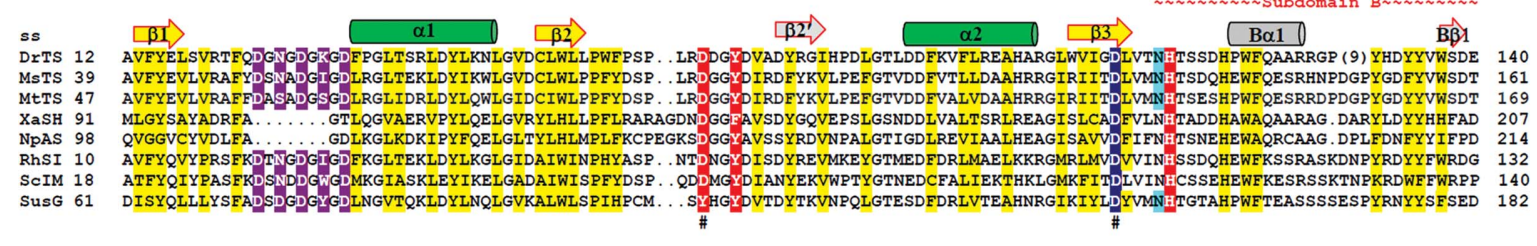

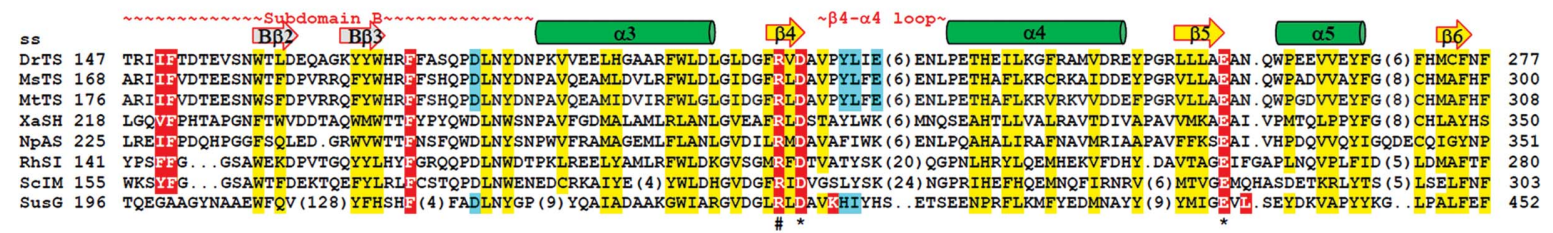

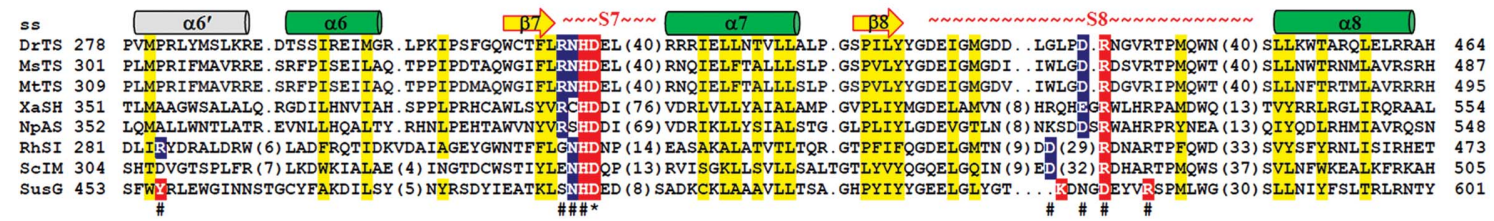
SUSG 453 SFWYRLEWGINNSTGCYFAKDILSY (5) NYRSDYIEATKLSNHDED (8) SADKCKLAAAVLLTSA. GHPYIYYGEELGLYGT... KDNGDEYVRSPMLWG (30) SLLNIYFSLTRLRNTY 601

Figure 3

(d)

Closest structural neighbours of DrTS. Structural superpositions of DrTS-N253A-Tris (red), XaSH-E322Q-sucrose (blue; PDB entry 3czk), NpASE328Q-maltoheptaose (green; PDB entry 1mw0) and RhSI-E254Q-sucrose (yellow; PDB entry 2pwe) for the $(\beta / \alpha)_{8}$ barrel $(a)$, subdomain B $(b)$ and domain $\mathrm{C}(c)$. These enzymes share a conserved $(\beta / \alpha)_{8}$ barrel, parts of subdomain B and a structurally conserved five-stranded sheet in domain $\mathrm{C}$, particularly the $\mathrm{C} \beta 2, \mathrm{C} \beta 3$ and $\mathrm{C} \beta 7$ strands. $(d)$ Structure-based sequence alignment of DrTS and its close homologues. Secondary-structure elements of DrTS are labelled and the numbers of residues in gaps are indicated in parentheses. BtSusG contains an additional carbohydrate-binding module inserted between $\mathrm{B} \beta 2$ and $\mathrm{B} \beta 3$. The residues of the $\mathrm{Mg}^{2+}$ and $\mathrm{Ca}^{2+}$ sites are shaded in magenta and cyan, respectively. The substrate-binding residues are shaded in red, whereas the residues of the conserved hydrophobic core are shaded in yellow. The catalytic triad residues are indicated with an asterisk, while the residues involved in stabilization of the triad orientation, but not in substrate binding, are shaded in blue and indicated with a hash, whereas those involved in both functions are shaded in red and indicated with a hash. 
dimeric MtTS may not be stable and a tetramer is thus formed with seven direct hydrogen bonds and ten salt bridges through subdomains B and S8. Oligomerization of the TS may enhance the structural stability, particularly in the loop-rich S8 and the two-stranded $\beta$-sheet of domain C. In monomeric GH13 members such as $\mathrm{XaSH}, \mathrm{NpAS}$ and RhSI, the two $\beta$-strands are more flexible and much shorter than those in TS (Fig. 3c), and are even absent in ScIM and sucrose phosphorylase (Yamamoto et al., 2010; Sprogøe et al., 2004). To explore the dimer function, the generation of monomeric DrTS mutants is being performed and their activity will be investigated.

\subsection{The similar active-site architecture}

The available crystal structures of GH13 enzymes in complex with substrates (or substrate analogues) display conserved active-site residues that form virtually identical interaction networks with the glucose residue at the -1 subsite

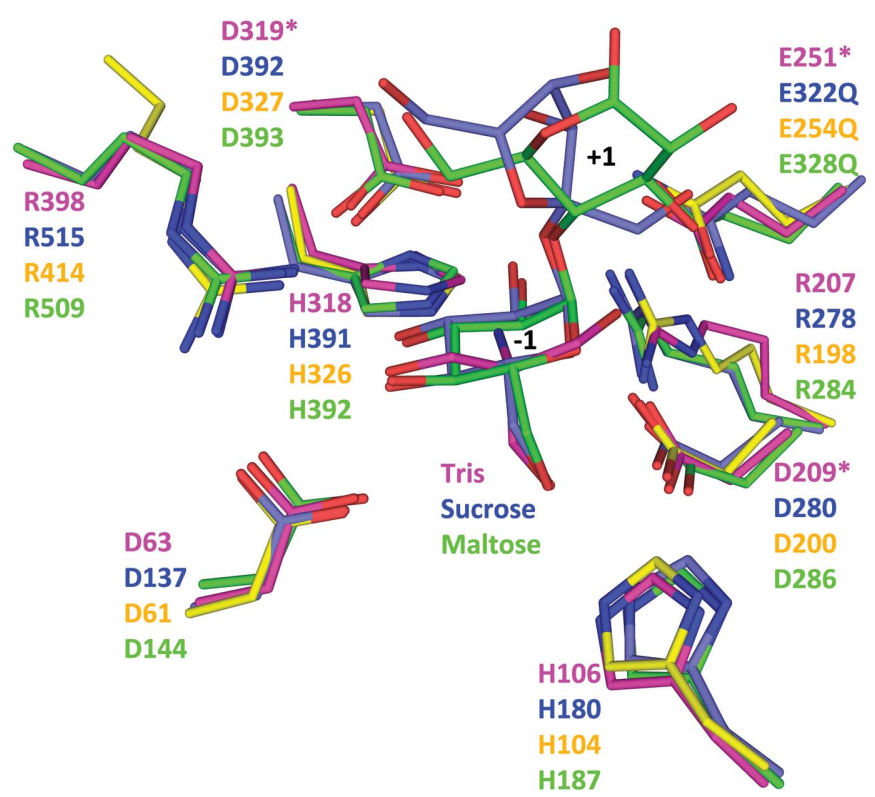

(a)

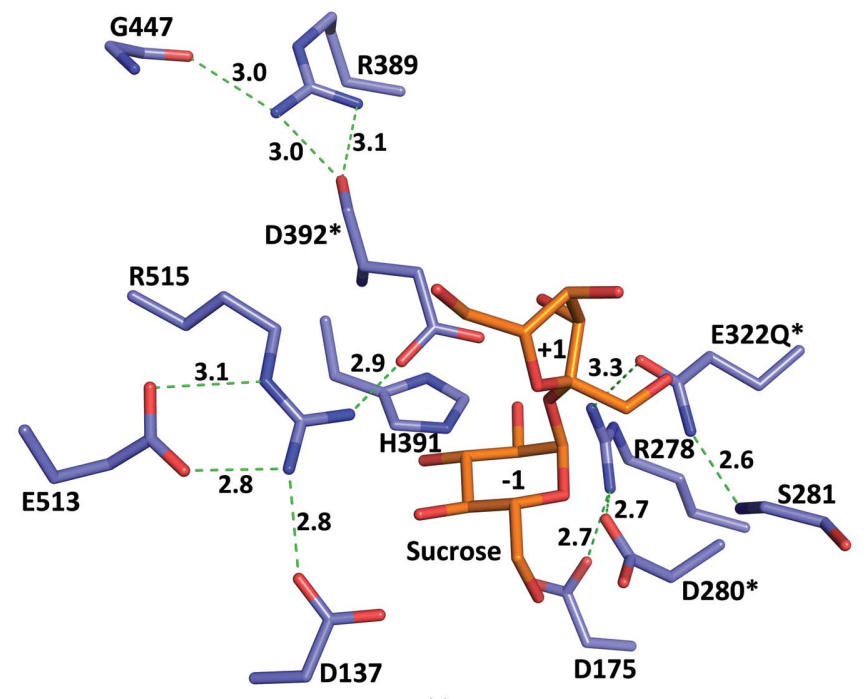

(c)

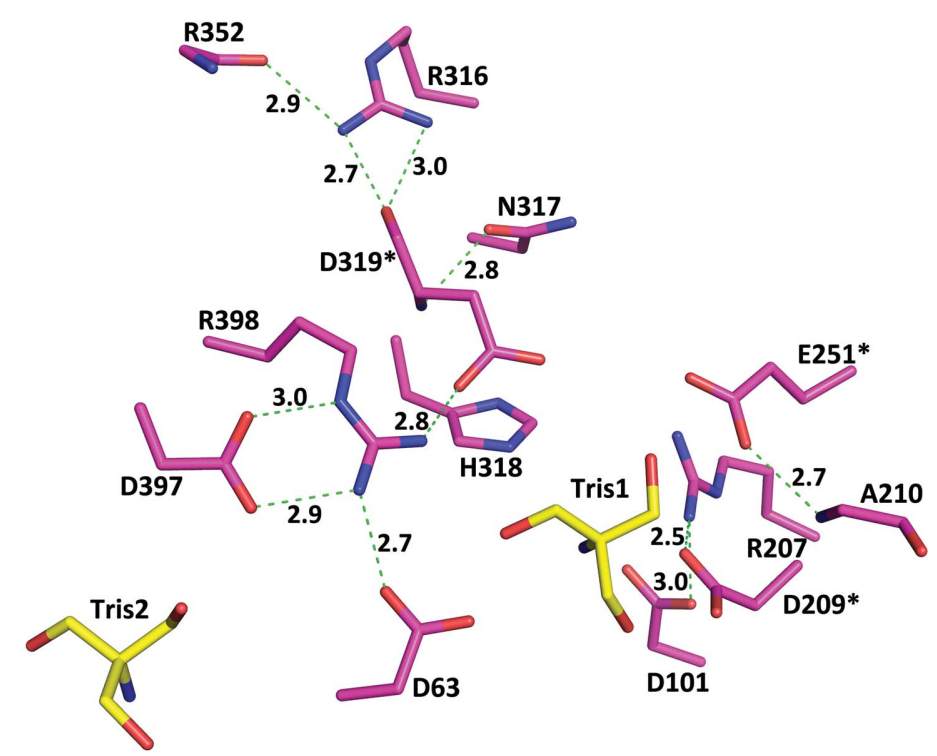

(b)

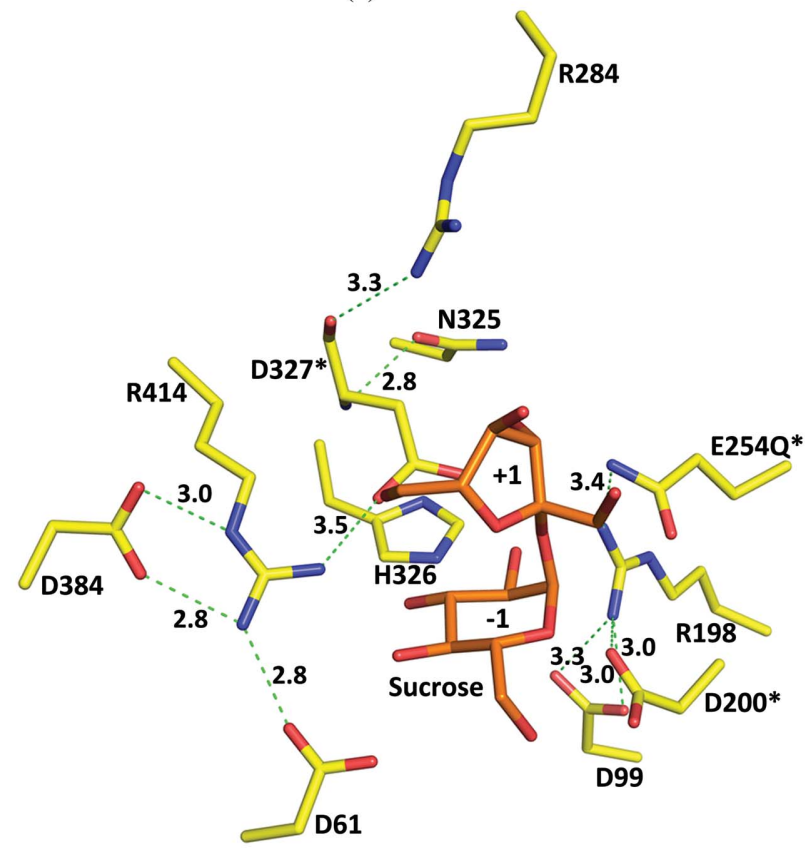

(d)

Figure 4

The similar active-site architecture. (a) Superposition of the -1 subsites of DrTS-N253A-Tris (megenta), XaSH-E322Q-sucrose (slate), NpAS-E328Qmaltoheptaose (green) and RhSI-E254Q-sucrose (yellow). The residue numbering is labelled in the same colour for each protein. The conserved activesite residues of DrTS occupy similar spatial positions to those of XaSH, NpAS and RhSI. Interestingly, the nonreducing terminal maltosyl residue in the NpAS-maltoheptaose complex fits the -1 and +1 subsites of DrTS well. $(b)-(d)$ The interaction networks between the catalytic triad and the surrounding residues in DrTS-N253A-Tris $(b)$, XaSH-E322Q-sucrose $(c)$ and RhSI-E254Q-sucrose $(d)$. The triad residues are indicated by an asterisk. 
(Kim et al., 2008; Skov et al., 2002; Ravaud et al., 2007; Yamamoto et al., 2011). These extensive interactions not only result in tight binding but also define the orientation of the nonreducing end to the -1 subsite for the double-displacement catalytic mechanism. A detailed structural comparison revealed that the active-site architecture of our DrTS structure resembles those of many other GH13 members such as its close structural matches (Fig. 4). The conserved active-site residues of DrTS occupy similar spatial positions and hence may form nearly identical interactions with the glucose residue at the -1 subsite upon substrate binding (Fig. $4 a$ ).

In addition, our comparison revealed that the conserved active-site residues form similar extensive interaction networks with the catalytic triad (Figs. $4 b, 4 c$ and $4 d$ ). Such interaction networks may be responsible for the attainment of the active conformation, as discussed below. In DrTS, the nucleophile Asp209 forms a salt bridge with Arg207, which also forms a salt bridge with Asp101, while the general acid/ base Glu251 forms a hydrogen bond to the carbonyl backbone of Ala210 (Fig. 4b). Interestingly, the transition-state stabilizer Asp319 forms a more extensive network with its surrounding residues. The carboxylate side chain of Asp319 stacks with His318 and forms a salt bridge with Arg398, which also forms salt bridges with Asp63 and Asp397. In addition, the carbonyl backbone of Asp319 forms a hydrogen bond to $\operatorname{Arg} 316$, which also hydrogen-bonds to the carbonyl backbone of Arg352. Virtually identical interaction networks are observed in $\mathrm{XaSH}$ and NpAS (Fig. 4c). Interestingly, structurally homologous but not sequence-equivalent $\mathrm{Arg}$ and Asp residues have been found to sustain the transition-state stabilizer Asp. For example, in RhSI the corresponding residue to Arg316 in DrTS is Gly324, and RhSI instead utilizes the first arginine (Arg284) in its unique isomerization motif to interact with the transition-state stabilizer Asp327 (Fig. 4d; Ravaud et al., 2007). Disruption of the interactions surrounding Asp327 in the randomly mutated $\mathrm{R} 284 \mathrm{C}$ variant with major hydrolytic activity causes a considerable change in subdomain orientations, leading to an open conformation (Lipski et al., 2013). In addition, the corresponding pair to Asp63-Arg398 in DrTS is absent in endo- $\alpha$-amylases, which instead use another conserved Asp-Arg pair (Asp545 and Arg549 in BtSusG) for both substrate binding and active-site stability (Fig. 3d; Koropatkin \& Smith, 2010; Janeček et al., 2014).

\subsection{The substrate-binding site}

In a number of GH13 enzymes, Tris has been shown to be a competitive inhibitor and is able to mimic a sugar molecule bound at the -1 subsite (Skov et al., 2002; Ravaud et al., 2007). An inhibitory effect of Tris on the activity of TS has also been observed (Filipkowski et al., 2012; Jiang et al., 2013). Our present assay results indicated that the activity of DrTS is inhibited strongly by Tris, with a $\mathrm{IC}_{50}$ value of $4.88 \mathrm{mM}$ in the presence of $50 \mathrm{~m} M$ maltose (Fig. 5). In the N253A structure at $2.21 \AA$ resolution, a strong electron-density peak was observed at the -1 subsite that correlates well with the shape of a Tris molecule (Fig. 6a). The peak was assigned as a Tris from the crystallization solution, which contained $300 \mathrm{mM}$ Tris- $\mathrm{HCl}$, forming hydrogen bonds to Asp63, His106, Asp209, Glu251 and Arg398. In the wild-type structure, a similar Tris signal was also observed. In the N253A mutant, an additional Tris-binding site was detected near to the -1 subsite, and the Tris molecule forms hydrogen bonds to two water molecules, the carboxylate group of Asp329 and the backbones of Leu61 and Arg62 (Fig. 6b).

Interestingly, in addition to the nearly identical active-site architecture of DrTS to those of XaSH and NpAS, the nonreducing terminal maltosyl residue in the NpAS-maltoheptaose complex fits the -1 and +1 subsites in DrTS-Tris well (Fig. 4a), and hence a maltose was modelled into the active site (Figs. $6 c$ and $6 d$ ). After modelling, energy minimization was performed in $C N S$ as a structural refinement (Brünger et al., 1998). The modelled DrTS-maltose structure suggested that Ile150, Ala210, Tyr213, Glu320 and Glu324 are involved in the +1 subsite (Fig. $6 c$ ). A conserved phenylalanine is present in the sucrose isomerases and therefore the $\mathrm{I} 150 \mathrm{~F}$ variant was constructed. $\mathrm{I} 150 \mathrm{~F}$ was found to display $\sim 55 \%$ of the isomerase activity and $\sim 170 \%$ of the hydrolase activity of the wild type. Thus, at this spatial position in DrTS an isoleucine is more favourable for maltose isomerization rather than a phenylalanine. However, neither isomerase nor hydrolase activity was detected when the Y213A, E320A and E324A mutants were examined, which indicates that these residues are essential for TS activity.

\subsection{Substrate-induced conformational changes}

The available crystal structures of SH, AS, SI and ScIM reveal that upon substrate binding the member-unique modules inserted within the $(\beta / \alpha)_{8}$ barrel move forward to cover and even to seal the active-site entrance (Fig. 7). A structural comparison of XaSH-sucrose and the apo $X$. campestris $\mathrm{SH}(\mathrm{XcSH})$ suggested that upon sucrose binding the $\mathrm{B} \beta 1-\mathrm{B} \beta 2$ loop becomes ordered and subdomains $\mathrm{B}$ and $\mathrm{S} 7$

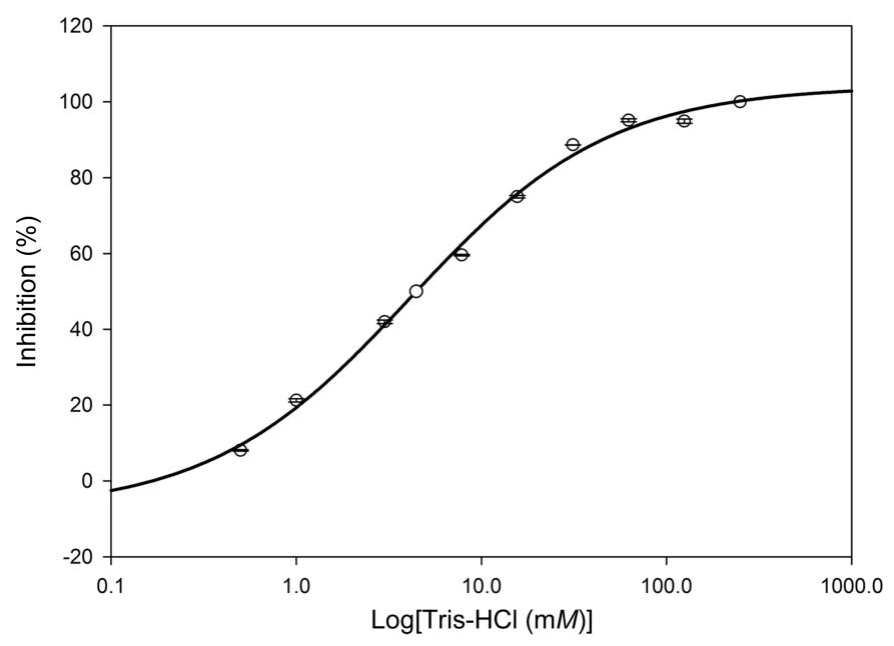

Figure 5

The inhibitory effect of Tris on the isomerase activity of DrTS. An $\mathrm{IC}_{50}$ value of $4.88 \mathrm{~m} M$ was estimated. All experiments were carried out in duplicate or triplicate. 
move forward to form the active conformation (Fig. 7a; Kim et al., 2008; Champion et al., 2009). In addition, the structures of $\mathrm{NpAS}$, with $36 \%$ sequence identity to $\mathrm{XaSH}$, display a similar conformation when in complex with Tris, glucose, sucrose and maltoheptaose (Skov et al., 2002). However, the apo $D$. radiodurans AS (DrAS) showed a strikingly different conformation in which the $\beta 2-\alpha 2$ loop is disordered and subdomains B and S7 swing away to create a more open active site (Skov et al., 2013). Moreover, the RhSI structures show a similar closed conformation when in complex with Tris, glucose, sucrose and inhibitors (Ravaud et al., 2007), whereas the R284C variant displays an open conformation with a drastic rearrangement of the SI-unique inserted modules, particularly subdomain B and the isomerization loop between $\beta 6$ and $\alpha 6$ (Fig. $7 b$; Lipski et al., 2013). The available ScIM structures display an almost closed conformation and the mechanism of substrate binding to the active site remains unclear (Yamamoto et al., 2010, 2011). Since ScIM shares 38\% sequence identity and high structural homology with RhSI, it is possible that subdomain $\mathrm{B}$ and its unique modules, particularly the $\beta 4-\alpha 4$ and $\beta 6-\alpha 6$ loops, may be involved in the opening and closing of the active site (Fig. $7 b$ ).

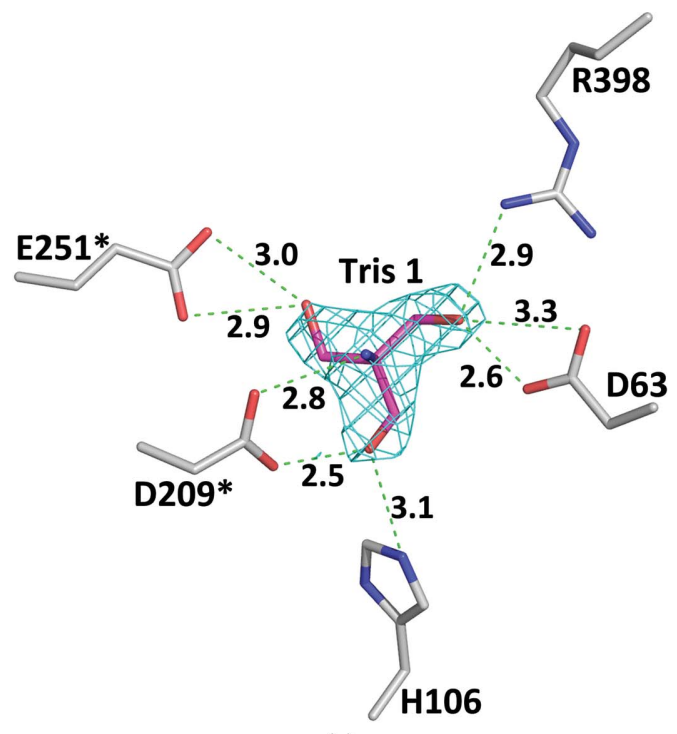

(a)

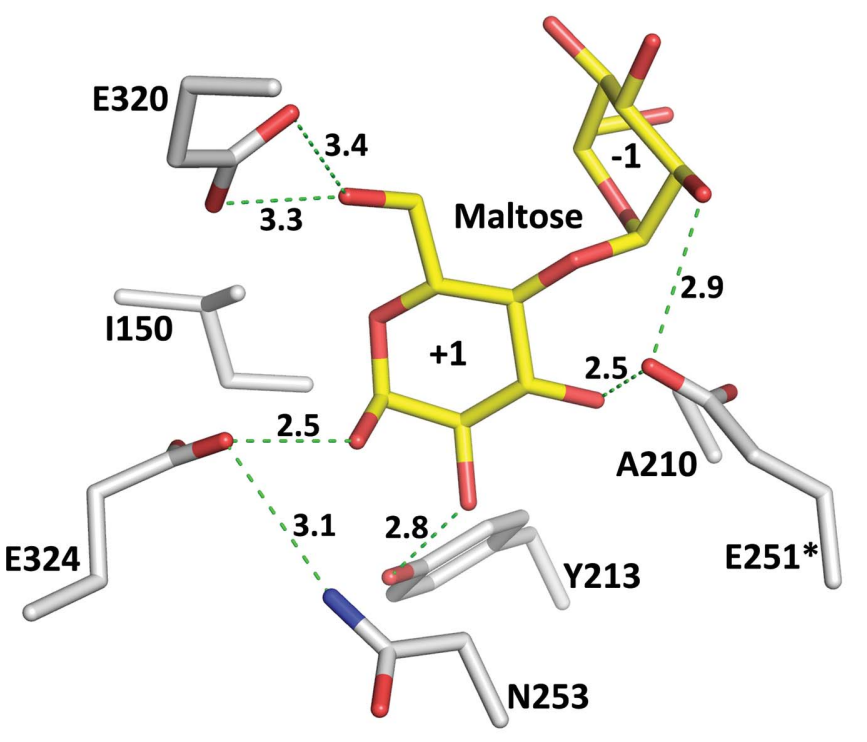

Figure 6

(c)

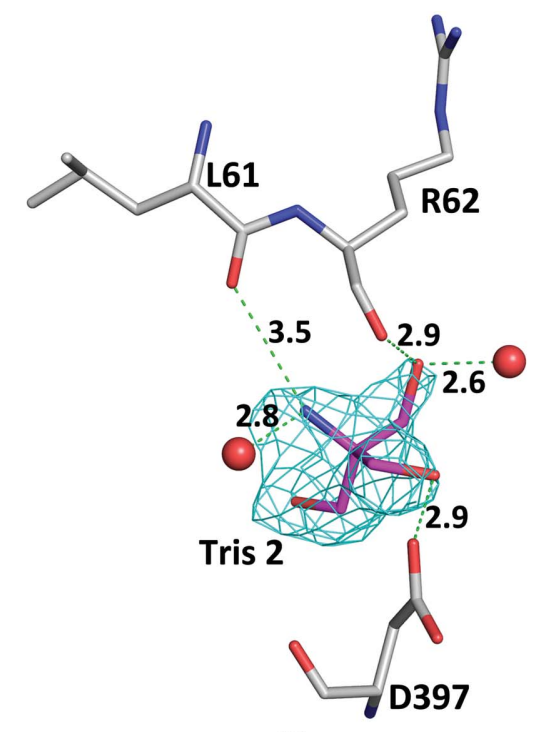

(b)

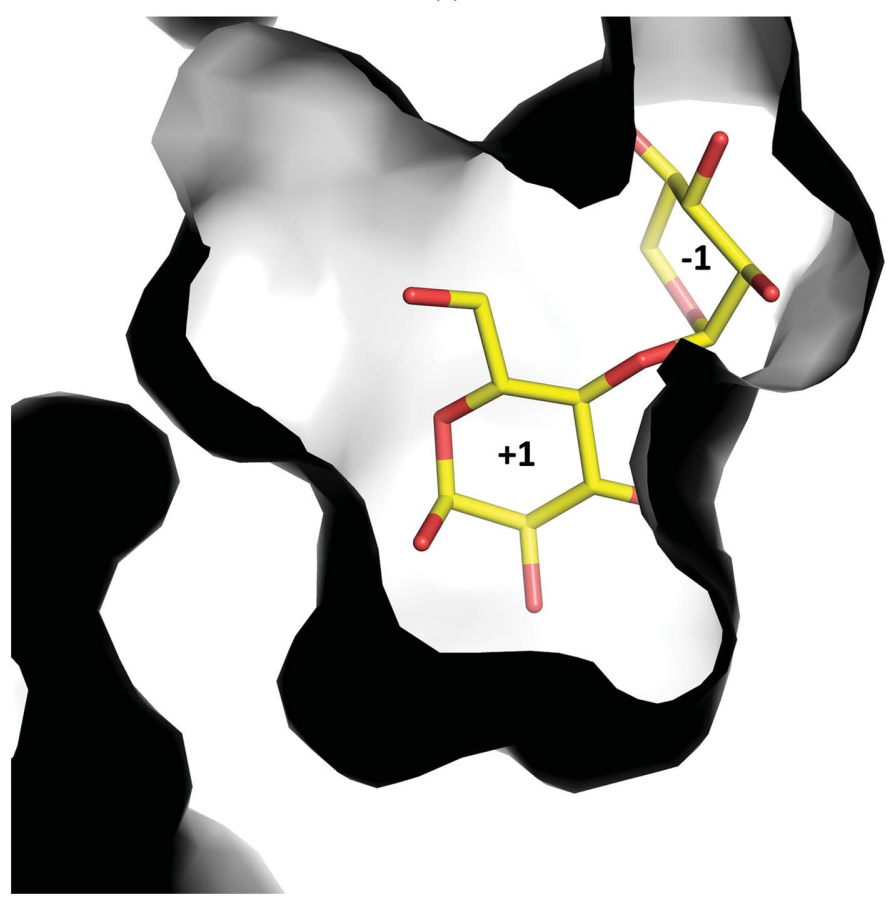

(d)

The substrate-binding site in DrTS. (a) The Tris bound at the -1 subsite in the N253A mutant. The simulated-annealing $F_{\mathrm{o}}-F_{\mathrm{c}}$ OMIT map for Tris is contoured at the $6 \sigma$ level. (b) An additional Tris-binding site in the N253A variant. The simulated-annealing $F_{\mathrm{o}}-F_{\mathrm{c}}$ OMIT map is contoured at the $4 \sigma$ level. $(c)$ The interaction networks between DrTS and the modelled maltose in the +1 subsite. Hydrogen bonds are shown as green dashed lines. $(d)$ Molecular surfaces of the +1 subsite. The view is the same as that in $(c)$. 
The crystal structures of DrTS, MsTS and MtTS display two significant structural differences (Fig. 7c). Firstly, the relative orientations between (sub)domains are different; in particular, subdomain B in MsTS and MtTS rotates away from the active site by $\sim 9^{\circ}$. Secondly, the tertiary structures of subdomain S7 are markedly distinct even if there is $>80 \%$ sequence identity between them. Interestingly, a similar rotation of subdomain B by $\sim 9^{\circ}$ and changes in S7 were also observed when substrate binds to $\mathrm{SH}$ and AS as described above. The structures of the the XaS-sucrose, NpAS-maltoheptaose and our DrTS-Tris complexes share a similar relative orientation of subdomain $\mathrm{B}$, particularly a similar spatial position of the $\mathrm{B} \beta 1-\mathrm{B} \beta 2$ loop (Fig. 7a), while those of apo XcSH, apo DrAS, MsTS and MtTS also display a similar subdomain B orientation. Because of high structural homology, in particular similar active-site architectures and subdomain B orientations of TS to $\mathrm{SH}, \mathrm{AS}$ and SI (Figs. 3, 4 and 7), we therefore conclude that the MsTS and MtTS structures may represent an open conformation of apo TS, while our DrTS-Tris complex represents a substrate-induced closed conformation for catalysis of the intramolecular isomerization.

\subsection{Open and closed TS conformations}

The crystal structure of MtTS showed two conformations (Roy et al., 2013). In one conformation, residues 353-381 in S7 are disordered and the active site is wide open to solvent (Figs. $7 c$ and $8 a$ ). On the other hand, the other MtTS structure is nearly identical to the MsTS structure (Fig. 7c). S7 is composed of two short $\alpha$-helices along with an extended loop, and the transition-state stabilizer Asp was shifted away from the active site by $\sim 4 \AA$ (Caner et al., 2013; Roy et al., 2013). In addition, Leu344 in MsTS, which is Leu352 in MtTS, projects into the active site and blocks the substrate-binding pocket (Fig. 8b). Compared with some GH13 members (Fig. 4), the active-site residues in the MsTS and MtTS structures adopt different spatial positions, and in addition the transition-state stabilizer Asp does not interact with its surrounding residues.

In our DrTS-Tris complex, the interaction networks formed between subdomains B, S7 and the active-site residues seal the active-site entrance (Fig. 8c). In particular, Arg148 forms salt

\section{Figure 7}

Different conformations observed in SH, SI and TS. Structural superposition of (a) XaSH-E322Q-sucrose (red), apo XcSH (green; PDB entry 2wpg) and DrTS-Tris (blue) and of (b) RhSI-E254Q-sucrose (red), the RhSI R284C mutant (green; PDB entry 4h2c) and ScIM-E277A-isomaltose (blue; PDB entry 3axh). Some of the member-unique insertions such as subdomains B and S7 in TS, SH and AS, or subdomains B and S and the segments between $\beta 4$ and $\alpha 4$ and between $\beta 6$ and $\alpha 6$ in SI and ScIM, modulate the size/shape and accessibility of the active site. These modules may move forward and backward from the active site to form different conformations during enzyme catalysis. (c) Stereoview of the structural superposition of DrTSTris, MsTS (PDB entry 3zoa) and MtTS (PDB entry 4lxf) chain $A$ and chain $B$. The subdomain B and S7 are shaded in blue, green, yellow, and red, respectively. These display distinct tertiary structures of S7 and the relative orientations of the subdomain $\mathrm{B}$ to the $(\beta / \alpha)_{8}$ barrel are different. A similar rearrangement of subdomain B was observed in TS, AS and SH. 
bridges with Glu223 and Glu324, which form hydrogen bonds to Gln254 and Asn253, respectively. In addition, Asp153 forms two hydrogen bonds to Asn347. In DrTS, Ile150, Phe151 and Phe173 in subdomain B and His318, Asp319, Glu320 and Glu324 in S7 all constitute part of the active-site pocket. This

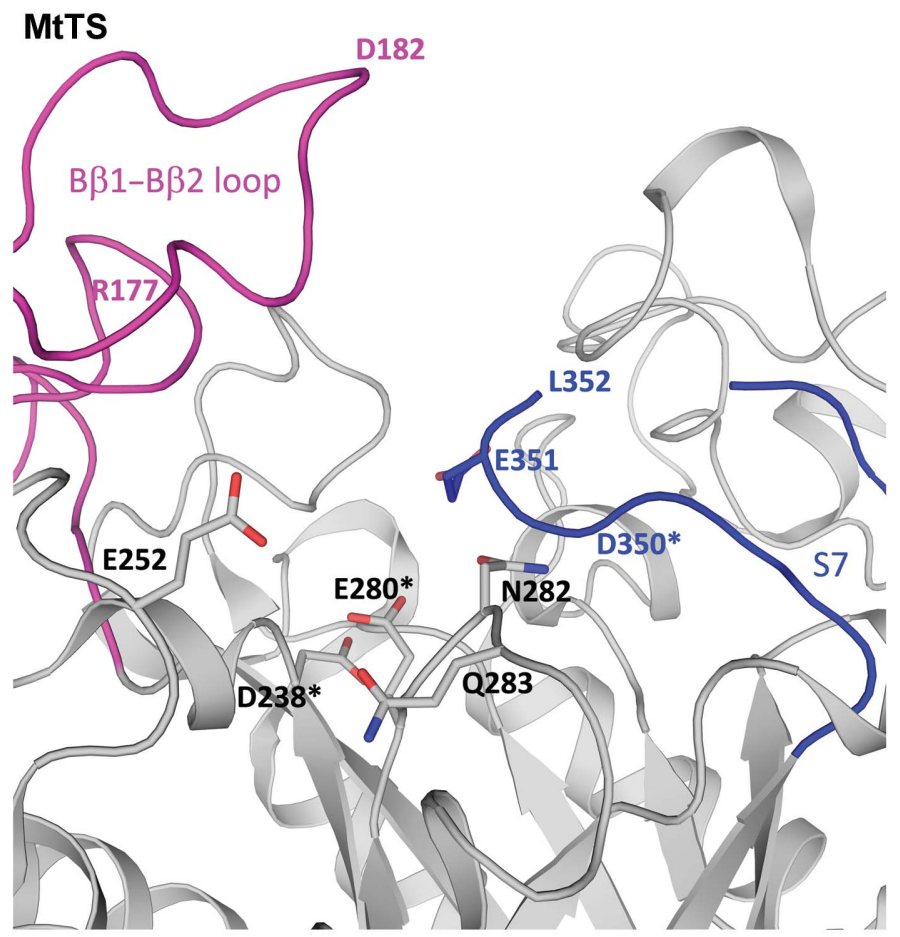

(a)

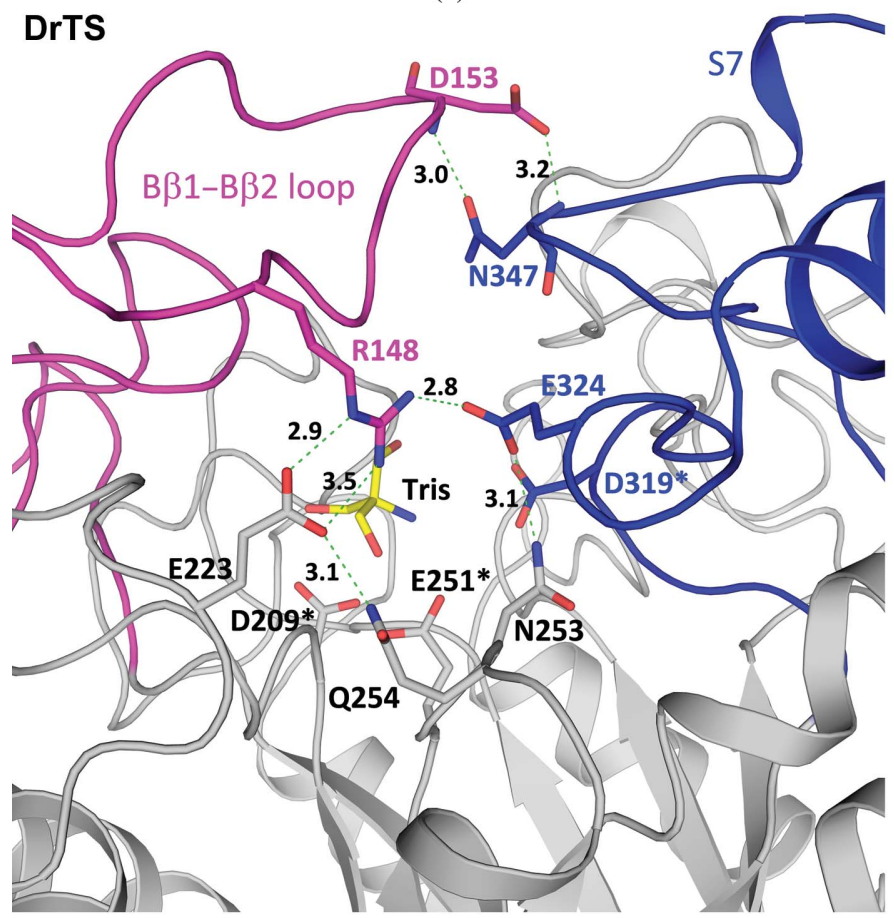

(c) means that interactions of the substrate with these residues would bring these two subdomains closer together to form a closed conformation. Such an enclosed active site in TS is needed for catalysis of the intramolecular isomerization reaction while minimizing hydrolysis of the sugar. The crystal

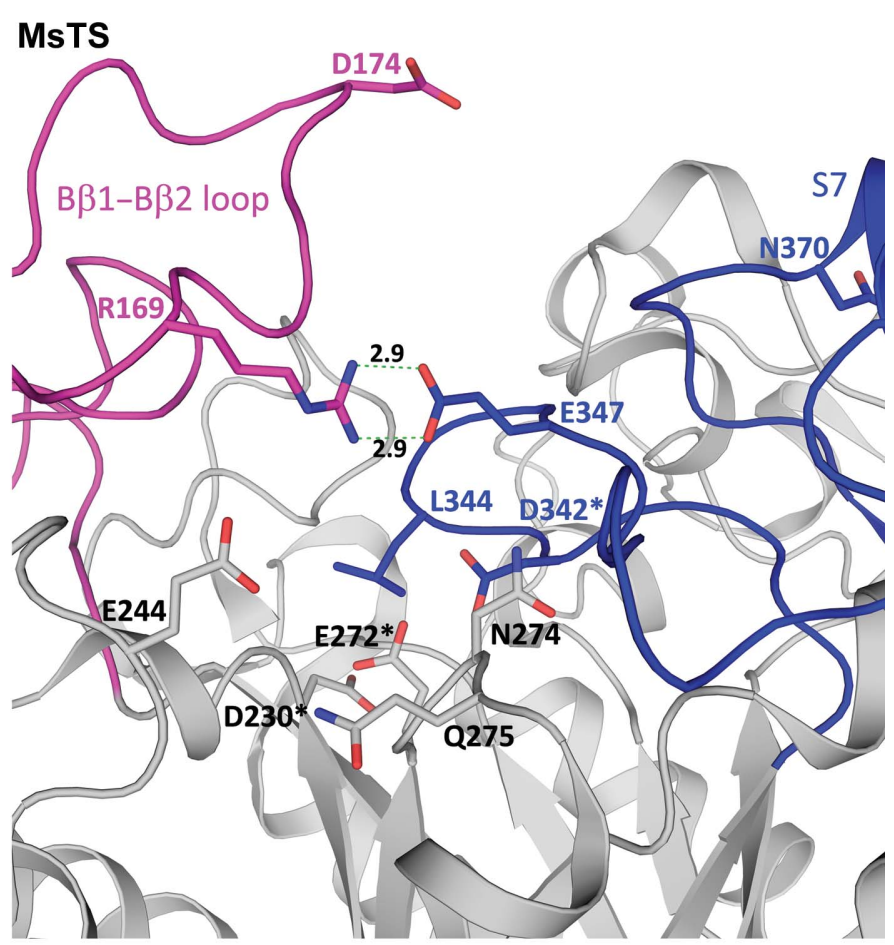

(b)

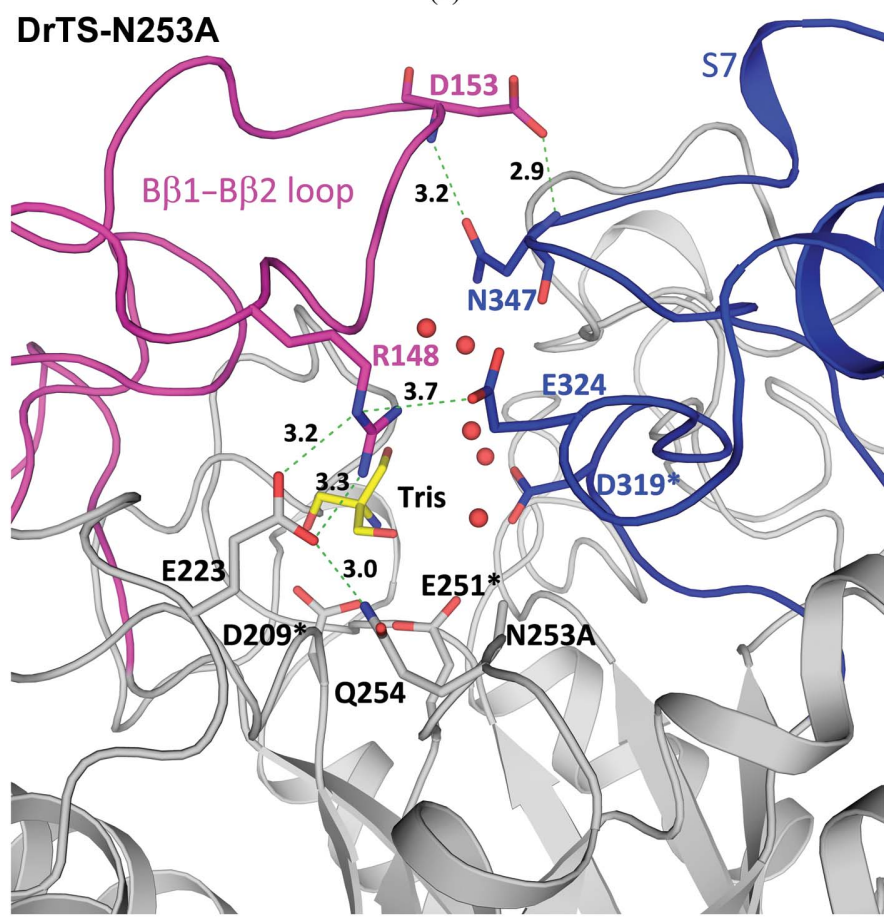

(d)

Figure 8

The interaction networks between subdomains B (magenta) and S7 (blue) in MtTS (PDB entry 4lxf; chain $B)(a)$, MsTS (b), DrTS-Tris $(c)$ and DrTSN253A-Tris $(d)$. In one MtTS structure the active site is wide open owing to a disordered region in S7, while in MsTS Leu344 blocks the substratebinding pocket. On the other hand, in DrTS a closed conformation was observed. These TS structures indicate that subdomains B and S7 serve as a gateway for the opening and closing of the active site. In N253A, the absence of the hydrogen bond between Asn253 and Glu324 causes movement of the Glu324 side chain, leading to the creation of a small pore for water entry. 
structures of the wild type and the N253A mutant are nearly identical, except for the side-chain orientation of Glu324 (Fig. $8 d$ ). In the N253A mutant, disruption of the interaction between Asn253 and Glu324 results in movement of the Glu324 side chain to create a small pore for solvent entry to the active site. This may explain why the N253A mutant has $\sim 11 \%$ of the isomerase activity and $\sim 180 \%$ of the hydrolase activity of the wild type. Similar to N253A, the R148A mutant shows $\sim 12 \%$ of the isomerase activity and $\sim 150 \%$ of the hydrolase activity of the wild type, suggesting that a small pore for water entry may also be present when Arg148 is replaced by alanine.

\subsection{Implications into the enzymatic mechanisms of TS}

Mechanistic analysis of MsTS demonstrated that the TScatalyzed isomerization of maltose to trehalose employs a twostep double-displacement mechanism (Zhang et al., 2011). The substrate binding allows subdomains B and S7 to move toward and seal the active-site pocket (Fig. 8c). Kinetic studies of MsTS suggested that such closure of the active site is the ratelimiting step. The nucleophile Asp attacks the anomeric centre of the glucose at the -1 subsite, yielding the covalent glycosylenzyme intermediate. The narrow and enclosed active site in DrTS and RhSI not only protects the glucosyl-enzyme intermediate from water, thus avoiding hydrolysis, but also allows the cleaved glucose/fructose at the +1 subsite to be retained to reorient and finally to re-attack the covalent intermediate in order to complete the isomerization reaction intramolecularly. Our present studies on DrTS will help to provide additional information that should aid in the modification of this enzyme for potential use in industrial applications.

We thank Dr R. Kirby for critical reading of the manuscript. This study was supported by the Ministry of Science and Technology (NSC102-2311-B-010-005 and NSC102-2313-B003-001) and by a grant from the Ministry of Education, Aim for the Top University Plan. Portions of this research were carried out at the National Synchrotron Radiation Research Center, a national user facility supported by the National Science Council of Taiwan, ROC. The Synchrotron Radiation Protein Crystallography Facility is supported by the National Core Facility Program for Biotechnology.

\section{References}

Brünger, A. T., Adams, P. D., Clore, G. M., DeLano, W. L., Gros, P., Grosse-Kunstleve, R. W., Jiang, J.-S., Kuszewski, J., Nilges, M., Pannu, N. S., Read, R. J., Rice, L. M., Simonson, T. \& Warren, G. L. (1998). Acta Cryst. D54, 905-921.

Caner, S., Nguyen, N., Aguda, A., Zhang, R., Pan, Y. T., Withers, S. G. \& Brayer, G. D. (2013). Glycobiology, 23, 1075-1083.

Cantarel, B. L., Coutinho, P. M., Rancurel, C., Bernard, T., Lombard, V. \& Henrissat, B. (2009). Nucleic Acids Res. 37, D233-D238.
Champion, E., Remaud-Simeon, M., Skov, L. K., Kastrup, J. S., Gajhede, M. \& Mirza, O. (2009). Acta Cryst. D65, 1309-1314.

Chen, V. B., Arendall, W. B., Headd, J. J., Keedy, D. A., Immormino, R. M., Kapral, G. J., Murray, L. W., Richardson, J. S. \& Richardson, D. C. (2010). Acta Cryst. D66, 12-21.

Christiansen, C., Abou Hachem, M., Janeček, Š., Viksø-Nielsen, A., Blennow, A. \& Svensson, B. (2009). FEBS J. 276, 5006-5029.

DeLano, W. L. (2002). PyMOL. http://www.pymol.org.

Elbein, A. D., Pan, Y. T., Pastuszak, I. \& Carroll, D. (2003). Glycobiology, 13, 17R-27R.

Emsley, P., Lohkamp, B., Scott, W. G. \& Cowtan, K. (2010). Acta Cryst. D66, 486-501.

Filipkowski, P., Pietrow, O., Panek, A. \& Synowiecki, J. (2012). Acta Biochim. Pol. 59, 425-431.

Holm, L. \& Rosenström, P. (2010). Nucleic Acids Res. 38, W545W549.

Hunter, R. L., Olsen, M. R., Jagannath, C. \& Actor, J. K. (2006). Ann. Clin. Lab. Sci. 36, 371-386.

Janeček, Š., Svensson, B. \& MacGregor, E. A. (2014). Cell. Mol. Life Sci. 71, 1149-1170.

Jiang, L., Lin, M., Zhang, Y., Li, Y., Xu, X., Li, S., Huang, H., Zhang, Y. \& Li, Y. (2013). PLoS One, 8, e77437.

Kim, M.-I., Kim, H.-S., Jung, J. \& Rhee, S. (2008). J. Mol. Biol. 380, 636-647.

Kobayashi, M., Hondoh, H., Mori, H., Saburi, W., Okuyama, M. \& Kimura, A. (2011). Biosci. Biotechnol. Biochem. 75, 15571563.

Koropatkin, N. M. \& Smith, T. J. (2010). Structure, 18, 200-215.

Lipski, A., Watzlawick, H., Ravaud, S., Robert, X., Rhimi, M., Haser, R., Mattes, R. \& Aghajari, N. (2013). Acta Cryst. D69, 298-307.

McCoy, A. J., Grosse-Kunstleve, R. W., Adams, P. D., Winn, M. D., Storoni, L. C. \& Read, R. J. (2007). J. Appl. Cryst. 40, 658-674.

Murshudov, G. N., Skubák, P., Lebedev, A. A., Pannu, N. S., Steiner, R. A., Nicholls, R. A., Winn, M. D., Long, F. \& Vagin, A. A. (2011). Acta Cryst. D67, 355-367.

Ravaud, S., Robert, X., Watzlawick, H., Haser, R., Mattes, R. \& Aghajari, N. (2007). J. Biol. Chem. 282, 28126-28136.

Roy, R., Usha, V., Kermani, A., Scott, D. J., Hyde, E. I., Besra, G. S., Alderwick, L. J. \& Fütterer, K. (2013). ACS Chem. Biol. 8, 22452255.

Šali, A. \& Blundell, T. L. (1993). J. Mol. Biol. 234, 779-815.

Schuck, P., Perugini, M. A., Gonzales, N. R., Howlett, G. J. \& Schubert, D. (2002). Biophys. J. 82, 1096-1111.

Skov, L. K., Mirza, O., Sprogøe, D., Dar, I., Remaud-Simeon, M., Albenne, C., Monsan, P. \& Gajhede, M. (2002). J. Biol. Chem. 277, 47741-47747.

Skov, L. K., Pizzut-Serin, S., Remaud-Simeon, M., Ernst, H. A., Gajhede, M. \& Mirza, O. (2013). Acta Cryst. F69, 973-978.

Sprogøe, D., van den Broek, L. A. M., Mirza, O., Kastrup, J. S., Voragen, A. G., Gajhede, M. \& Skov, L. K. (2004). Biochemistry, 43, 1156-1162.

Stam, M. R., Danchin, E. G. J., Rancurel, C., Coutinho, P. M. \& Henrissat, B. (2006). Protein Eng. Des. Sel. 19, 555-562.

Svensson, B. (1988). FEBS Lett. 230, 72-76.

Wang, J.-H., Tsai, M.-Y., Chen, J.-J., Lee, G.-C. \& Shaw, J.-F. (2007). J. Agric. Food Chem. 55, 3435-3443.

Yamamoto, K., Miyake, H., Kusunoki, M. \& Osaki, S. (2010). FEBS J. 277, 4205-4214.

Yamamoto, K., Miyake, H., Kusunoki, M. \& Osaki, S. (2011). J. Biosci. Bioeng. 112, 545-550.

Zhang, R., Pan, Y. T., He, S., Lam, M., Brayer, G. D., Elbein, A. D. \& Withers, S. G. (2011). J. Biol. Chem. 286, 35601-35609. 